\title{
XFEM/LEVEL-SET FOR MODELLING OF COATED INCLUSION COMPOSITES
}

\author{
Tran Anh Binh \\ National University of Civil Engineering, Hanoi, Vietnam \\ E-mail: anh-binh.tran@nuce.edu.vn
}

Received July 20, 2016

\begin{abstract}
In this paper, Extended Finite Element method (XFEM) is used to model the embedded coated inclusion composite. The coated inclusion with finite thickness is associated with two level-set functions, which describe its inside and outside interfaces. A simple integration rule is employed for numerical quadrature in elements cut by two interfaces. Accuracy and efficiency of the proposed approach are demonstrated through 3D numerical examples and applied to homogenization of such materials.
\end{abstract}

Keywords: XFEM, multiple level-sets, coated inclusion.

\section{INTRODUCTION}

In composite engineering problems, thin coatings are often placed between a matrix and an inclusion and show the important role in the effective behavior of a matrixinclusion composites [1,2]. A large number of models of this type based on the framework of micromechanics have been developed in recent years. Some theoretical and experimental models are studied for inclusions with spherical and cylindrical shapes [3-5] and for ellipsoidal coated inclusion [6-9]. These studies are limited in the case of simple inclusion shapes. Numerical techniques allow overcoming these issues but efficient strategies must be developed to avoid important computational costs, especially in the case of complex three-dimensional (3D) models. Most of numerical approaches are based on finite element method [10-13]. It requires sophisticated 3D mesh softwares, and large computational times related to the meshing operation. Another way is to use the Fast Fourier Transform method (FFT) without meshing to calculate effective properties [14,15]. However, a good convergence is only reached with large iterations in the case of high contrast of matrix-inclusion properties. More recently, XFEM method has been proposed for modelling and computing the overall properties of embedded coated inclusion composite. A major advantage is to describe the interfaces implicitly through a level set function, with an enriched approximation of the finite element scheme to add different jumps at the interfaces. While Benvenuti [16] simulates the coating as an inclusion

(C) 2017 Vietnam Academy of Science and Technology 
with an equivalent eigenstrain within XFEM scheme. The studies of Julien, Benvenuti, $\mathrm{Zhu}, \mathrm{Gu}$ [17-20] treat also the thin coatings as imperfect interfaces. This paper proposes simple way to model the coated inclusion with help of two level-set functions, which describe two interfaces bounding the coating. Additional degrees of freedom (d.o.f.) are introduced for nodes whose support is cut by more than one interface. The layout of this paper is as follows.

First, the proposed approach is presented in section 2. In the next section, the effective conductivity of matrix composite with spherical or arbitrary coated inclusions is numerically investigated. Some conclusions is finally pointed out in the last section.

\section{XFEM/LEVEL-SET FOR MODELLING OF COATED INCLUSION COMPOSITES}

Modeling a domain containing interfaces with the finite Element Method (FEM) requires a mesh conforming with all internal surfaces. This operation may take large computational time for complex three-dimensional geometries. In contrast, the XFEM overcomes these issues by enriching the finite element approximation with additional functions to model interfaces independently of the background mesh.

Consider a domain $\Omega \subset \mathbb{R}^{D}$ ( $D$ being the dimension of the domain) which is partitioned into finite elements. The XFEM displacement approximation takes the form [21]

$$
T^{h}(\mathbf{x})=\sum_{i \in \mathcal{S}} N_{i}(\mathbf{x}) T_{i}+\sum_{k}^{n_{\text {int }}} \sum_{j \in \mathcal{S}^{e k}} N_{j}(\mathbf{x}) \psi^{k}\left(\phi^{k}(\mathbf{x})\right) a_{j}^{k} .
$$

In the above equation, $T^{h}$ is temperature field; $a_{j}^{k}$ are nodal unknowns. $n_{\text {int }}$ is number of interfaces of coated inclusions. In this paper, we assume $n_{\text {int }}=2$, corresponding to inside interface $\Gamma_{1}$ and outside interface $\Gamma_{2}$ of coated inclusion (see Fig. 1). The proposed method can be used for modelling of multi-coated inclusion, i.e $n_{\text {int }}>2$. The nodal set $\mathcal{S}^{e k}$ is defined as

$$
\mathcal{S}^{e k}=\left\{j \mid j \in \mathcal{S}, \omega_{j} \cap \Gamma^{k} \neq 0\right\},
$$

where $\mathcal{S} \subset \mathbb{N}^{*}\left(\mathbb{N}^{*}\right)$ being the set of positive natural numbers) be a set of $N$ nodes indices in the mesh; $\omega_{i}=\operatorname{supp}\left(n_{i}\right)$ being the support of the nodal shape function $N_{i}$, which

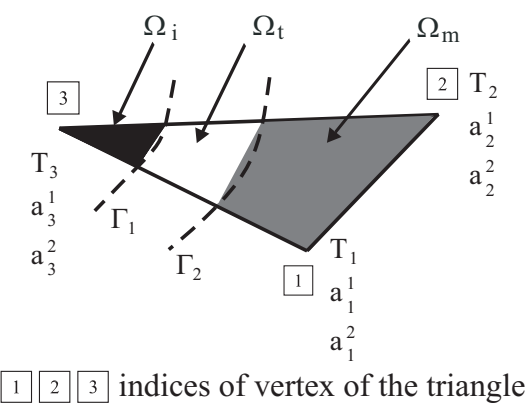

Fig. 1. Nodal unknowns at node for an element cut by both inside and outside interfaces of coated inclusions 
consists of the union of all elements connected to the node $n_{i} \cdot \psi^{k}\left(\phi^{k}(\mathbf{x})\right)$ is an enrichment function constructed via the level-set function $\phi^{k}$ of inclusion $k$ with interface $\Gamma^{k}$

$$
\psi^{k}\left(\phi^{k}(\mathbf{x})\right)=\sum_{i}\left|\phi_{i}^{k}\right| N_{i}(\mathbf{x})-\left|\sum_{i} \phi_{i}^{k} N_{i}(\mathbf{x})\right| .
$$

The general level-set function $\phi^{k}$ takes the form

$$
\Gamma_{k}=\left\{\mathbf{x} \in \mathbb{R}^{d} \mid \phi^{k}(\mathbf{x})=0\right\} .
$$

We can opt to choose the signed distance function for $\phi^{k}(\mathbf{x})$, which is given in [21]. For a spherical coated inclusion, $\phi^{k}(\mathbf{x})$ is expressed by

$$
\phi^{k}(\mathbf{x})=\min \left\{\left\|\mathbf{x}-\mathbf{x}_{c, i}^{k}\right\|-r_{i}^{k}\right\}, \quad i=1,2, \ldots, n_{c}
$$

with $k=1,2 ; \phi^{1}(\mathbf{x}), \phi^{2}(\mathbf{x})$ being level-set function which describe inside and outside interfaces of coated inclusions, respectively. $\mathbf{x}_{c, i}^{1}, \mathbf{x}_{c, i}^{2}$ are centers of inside interface and outside interface of coated inclusion $i . r_{i}^{1}, r_{i}^{2}$ are inside and outside radii of coated inclusion $i$. We can model arbitrary inclusion shapes using level-set function defined in [20]. With helps of function $\phi^{k}(\mathbf{x})$, we can easily to divide the domain $\Omega$ into 3 subdomains $\Omega=\bigcup\left(\Omega_{i}, \Omega_{m}, \Omega_{t}\right)$ in which $\Omega_{i}, \Omega_{m}, \Omega_{t}$ are defined as (see Fig. 2)

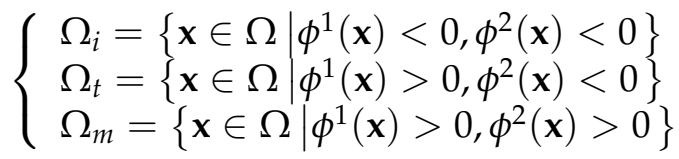

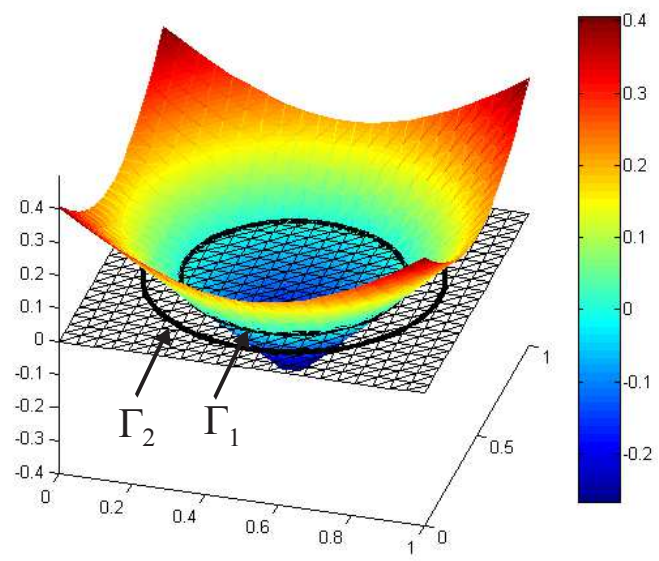

(a)

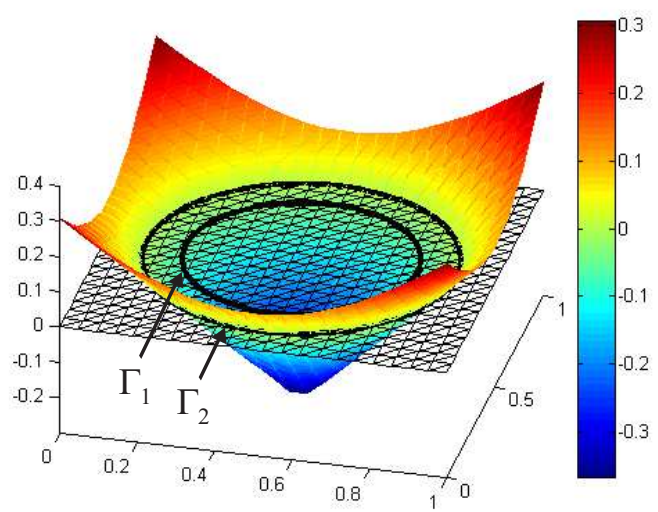

(b)

Fig. 2. Level-set functions of a) $\Gamma_{1}$ and b) $\Gamma_{2}$ of circular coated inclusion

The enriched approximation (1) adds one (or two) additional d.o.fs for node to describe one (or two) discontinuities presented in a single element, as depicted schematically in Fig. 1. This technique induces the difficulty on the numerical integration over an element cut by both inside and outside interfaces. This difficulty relates to the numerical 


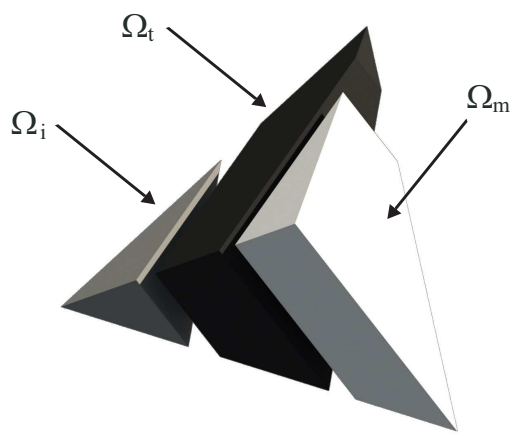

Fig. 3. A tetrahedral element cut by both inside and outside interfaces and partitioning of the element domain into subdomains $\Omega_{i}, \Omega_{t}$ and $\Omega_{m}$

integration on the domain $\Omega_{t}$ as illustrated in Fig. 3. To overcome this issue, we propose a simple integration rule. The idea is to decompose the integration in domain $\Omega$ into subdomains $\Omega_{i}$ using a Gauss quadrature rule.

$$
f(\mathbf{x})= \begin{cases}f_{i}(\mathbf{x}), & \text { if } \mathbf{x} \in \Omega_{i} \\ f_{t}(\mathbf{x}), & \text { if } \mathbf{x} \in \Omega_{t} \\ f_{m}(\mathbf{x}), & \text { if } \mathbf{x} \in \Omega_{m}\end{cases}
$$

where the function $f_{i}(\mathbf{x}), f_{t}(\mathbf{x})$ and $f_{m}(\mathbf{x})$ are associated with the inclusions, interfacial domain and matrix materials, respectively. We can decompose this integration into

$$
\int_{\Omega} f(\mathbf{x}) d \Omega=\int_{\Omega_{t}} f_{t}(\mathbf{x}) d \Omega+\int_{\Omega_{m}} f_{m}(\mathbf{x}) d \Omega+\int_{\Omega_{i}} f_{i}(\mathbf{x}) d \Omega .
$$

To overcome numerical difficulties related to the integration of $f_{t}(\mathbf{x})$ over the domain $\Omega_{t}$, we use a simple superposition technique as follows

$$
\int_{\Omega_{t}} f_{t}(\mathbf{x}) d \Omega=\int_{\Omega} f_{t}(\mathbf{x}) d \Omega-\int_{\Omega_{m}} f_{t}(\mathbf{x}) d \Omega+\int_{\Omega_{i}} f_{t}(\mathbf{x}) d \Omega,
$$

where $f_{t}: \Omega \subset \mathbb{R}^{d} \rightarrow \mathbb{R}$ is extended over the domain $\Omega$.

By introducing the above formula into Eq. (8), the integration of $f(x)$ over the tetrahedral domain is expressed as

$$
\int_{\Omega} f(\mathbf{x}) d \Omega=\int_{\Omega} f_{t}(\mathbf{x}) d \Omega+\int_{\Omega_{i}}\left(f_{i}(\mathbf{x})-f_{t}(\mathbf{x})\right) d \Omega+\int_{\Omega_{m}}\left(f_{m}(\mathbf{x})-f_{t}(\mathbf{x})\right) d \Omega .
$$

All integrations of right-hand Eq. (10) can be easily calculated, as $\Omega_{m}\left(\Omega_{i}\right)$ are composed of only a few tetrahedra. Then it only requires: (i) constructing intersections between the element $\Omega^{e}$ and the linear approximations of level-set functions $\phi^{k}$; (ii) partitioning the domains $\Omega_{m}\left(\Omega_{i}\right)$ into tetrahedra. The intersection points $\mathbf{x}_{p}$ are given by

$$
\mathbf{x}_{p}=\mathbf{x}_{i}+\xi\left(\mathbf{x}_{j}-\mathbf{x}_{i}\right), \xi=-\frac{\phi_{i}^{k}}{\phi_{j}^{k}-\phi_{i}^{k}},
$$




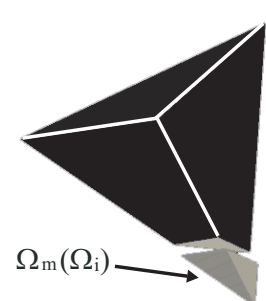

(a)



(b)

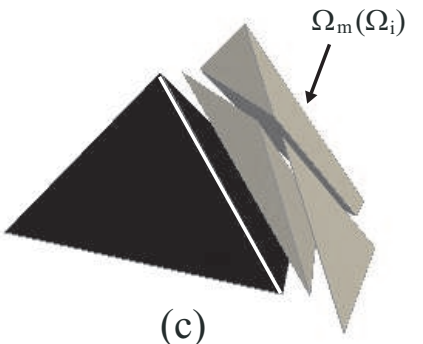

(c)

Fig. 4. Three possibilities for subdivision of subdomains $\Omega_{m}\left(\Omega_{i}\right)$ into tetrahedra

where $\mathbf{x}_{j}$ are two edge points of an element, $\phi_{i}^{k}$ and $\phi_{j}^{k}$ are values of the level-set function of interface $\Gamma_{k}$ at the points $\mathbf{x}_{i}$, and $\mathbf{x}_{j}$. Once the domains $\Omega_{m}\left(\Omega_{i}\right)$ are constructed, the subdivision of $\Omega_{m}\left(\Omega_{i}\right)$ into tetrahedra is easily obtained as illustrated in Fig. 4 . The gray tetrahedra belong to domains $\Omega_{m}\left(\Omega_{i}\right)$.

\section{NUMERICAL EXAMPLES FOR EFFECTIVE CONDUCTIVITY}

The field equations of thermo-static problem are given by

$$
\begin{aligned}
& \nabla \cdot \mathbf{q}(\mathbf{x})-r(\mathbf{x})=0 \text { in } \Omega, \\
& \mathbf{q}(\mathbf{x})=-C(\mathbf{x}) \nabla T(\mathbf{x}) .
\end{aligned}
$$

Above, $\mathbf{q}(\mathbf{x})$ denotes the heat flux, $r(\mathbf{x})$ is a heat source term and $c(\mathbf{x})$ the conductivity tensor. $C(\mathbf{x})$ is local conductivity and given by

$$
C(\mathbf{x})= \begin{cases}C_{i}(\mathbf{x}), & \text { if } \mathbf{x} \in \Omega_{i} \\ C_{t}(\mathbf{x}), & \text { if } \mathbf{x} \in \Omega_{t} \\ C_{m}(\mathbf{x}), & \text { if } \mathbf{x} \in \Omega_{m}\end{cases}
$$
equations

Using XFEM/Level-set procedure, one finds the discrete system of linear ordinary

$$
\mathbf{K D}=\mathbf{Q}, \quad \mathbf{D}=\left\{\begin{array}{ll}
\mathbf{T} & \mathbf{a}
\end{array}\right\}^{\mathrm{T}},
$$

where $\mathbf{D}$ are nodal unknowns, $\mathbf{K}$ and $\mathbf{Q}$ are the global stiffness matrix and external flux, respectively. More precisely, the matrix $\mathbf{K}$ and vector $\mathbf{Q}$ are defined by

$$
\mathbf{K}=\int_{\Omega} \mathbf{B}^{T} C(\mathbf{x}) \mathbf{B} d \Omega, \quad \mathbf{Q}=\int_{\Omega} \mathbf{N}^{\mathrm{T}} \mathrm{r} d \Omega,
$$

where $\mathbf{B}$ and $\mathbf{N}$ are the matrices of shape function derivatives and shape functions associated with the approximation scheme 1.

As the first example, we consider a spherical coated inclusion of inside radius $r_{1}$ and exterior radius $r_{2}$, embedded in a cubic box of $L \times L \times L$ dimension (see Fig. 5(a)). The effective conductivity of composite $c^{e f f}$ is computed by using proposed approach. A 
convergence analysis is carried out, using different meshes with increasing nodal densities associated with a characteristic element size $h$. This spherical composite is submitted to a homogeneous temperature gradient field

$$
T=\bar{T}=-\mathbf{e}_{0} \mathbf{x},
$$

on its boundary with $\mathbf{e}_{\mathbf{0}}$ being a constant vector. The effective conductivity is given by the following relationship

$$
\langle\mathbf{q}(\mathbf{x})\rangle_{\Omega}=-c^{e f f} \nabla\langle T(\mathbf{x})\rangle_{\Omega}
$$

where $\langle\bullet\rangle$ denotes the volume average on $\Omega$ of an object. We note that with the boundary condition (17), we have $\langle T(\mathbf{x})\rangle_{\Omega}=-\mathbf{e}_{0}$. Once we have temperature field, the left-handside of Eq. (18) can be computed as follows

$$
\langle\mathbf{q}(\mathbf{x})\rangle_{\Omega}=\frac{1}{V} \int_{\Omega} C(\mathbf{x}) \nabla T(\mathbf{x}) d \Omega,
$$

where $V$ is volume of domain $\Omega$.

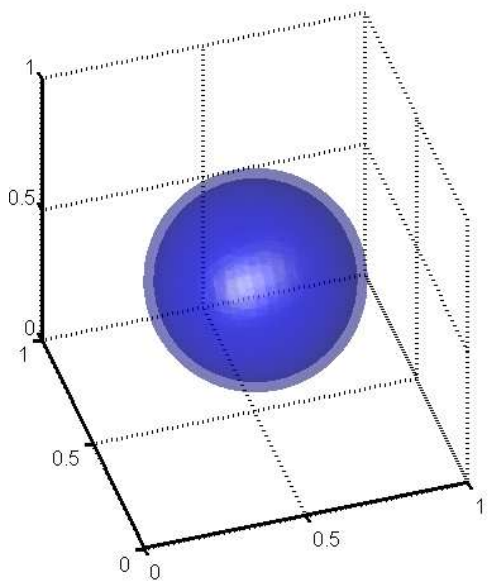

(a)

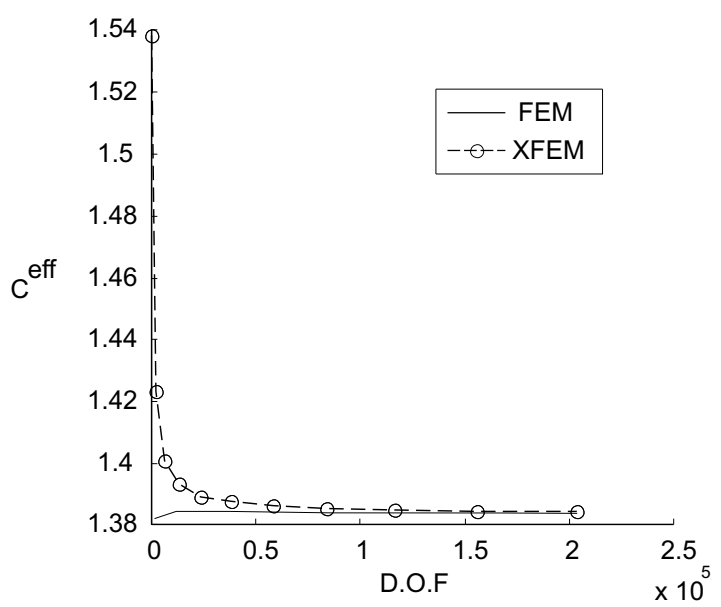

(b)

Fig. 5. a) A spherical coated inclusion embedded in a cubic box; b) Convergence of the effective conductivity versus the total number of d.o.f.

To test our numerical procedure, we compare the XFEM solution and the FEM one with fine mesh. The cubic domain is meshed with tetrahedra for both FEM and XFEM method. While the FEM method use a mesh conforming with spherical interface, the XFEM method use a regular mesh to define implicitly spherical inclusion. Numerical parameters are taken as follows: $C_{m}=1 \mathrm{Wm}^{-1} \mathrm{~K}^{-1}, C_{t}=10 \mathrm{Wm}^{-1} \mathrm{~K}^{-1}, C_{i}=100$ $\mathrm{Wm}^{-1} \mathrm{~K}^{-1}, r_{1}=0.3 L, r_{2}=0.33 \mathrm{~L}$. The effective conductivity of spherical composite is plotted in Fig. 5(b). An excellent agreement between the XFEM solution and the FEM one is obtained. 
We now change the radius of sphere to investigate effects of inclusion volume fraction $V_{i}$ on the effective conductivity. By using full our computer resource, the result of XFEM, FEM and FFT [15] are shown in Fig. 6. The XFEM method seem to give better result then FFT method as compared as FEM method.

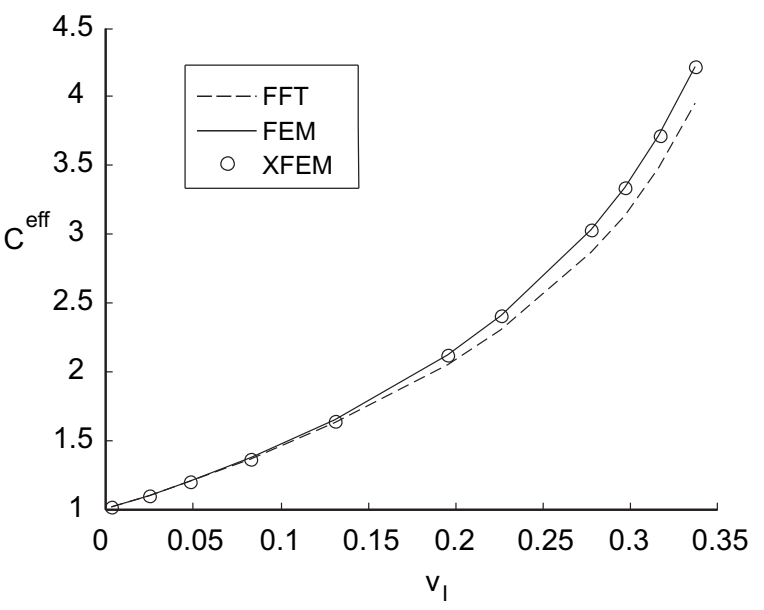

Fig. 6. Effect of inclusion volume fraction on the effective conductivity

In the second example, we consider a random spherical coated composite as depicted in Fig. 7. The composite contain 40 random inclusions with minimal and maximal exterior radii $r_{2}^{\max }=0.3250 \mathrm{~L}$ and $r_{2}^{\min }=0.0859 \mathrm{~L} ; \mathrm{L}$ being the dimension of cubic. The interior radii are taken as $r_{1}=0.85 r_{2}$. The minimum distance between coated inclusions is $0.0398 \mathrm{~L}$. The total volume fraction of inclusions, coating domain and matrix are $V_{i}=0.2228, V_{t}=0.1331$ and $V_{m}=0.6441$, respectively. The purpose of this example is to compute the effective conductivity associated with the homogenized material. The material parameters are taken the same as previous example. The convergence analysis of results is shown in Fig. 8. A good agreement between FEM and XFEM is observed. These

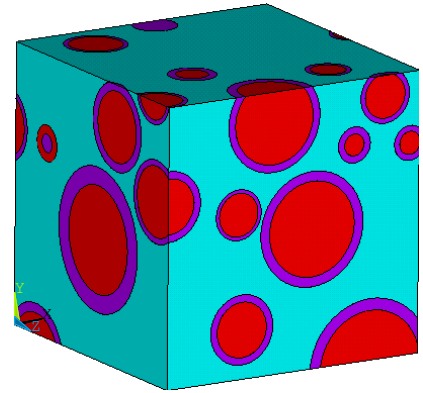

(a)



(b)

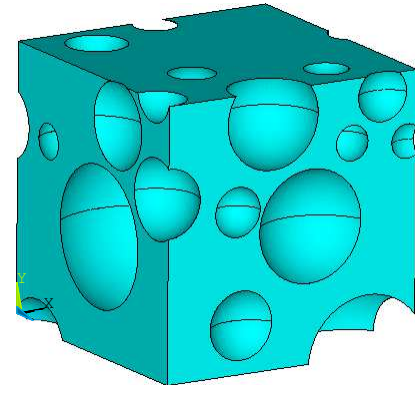

(c)

Fig. 7. a) Cubic containing 40 random spherical coated inclusions;

b) 40 coated inclusions of cubic, c) matrix domain of cubic 




Fig. 8. Convergence of the effective conductivity versus the total number of d.o.f.

results are obtained with help a super computer of 400GB RAM and 32 cores of LMT lab Cachan University - France. For using the full computational power of the machine, we code the program in MATLAB with parallel technique to take advantage of multicore and multiprocessor computers.

We can notice that the FEM method converges faster than proposed method but it is not easy to obtain the conforming mesh of FEM with complex microstructure. The computational cost using XFEM on Matlab is higher than FEM when the conforming mesh of FEM is provided. The computational time of FEM contain two parts: time to create the conforming mesh in Ansys software and time to run code on Matlab platform. This is illustrated by a recent statement of Ted Blacker, manager of simulation sciences at Sandia



(a)

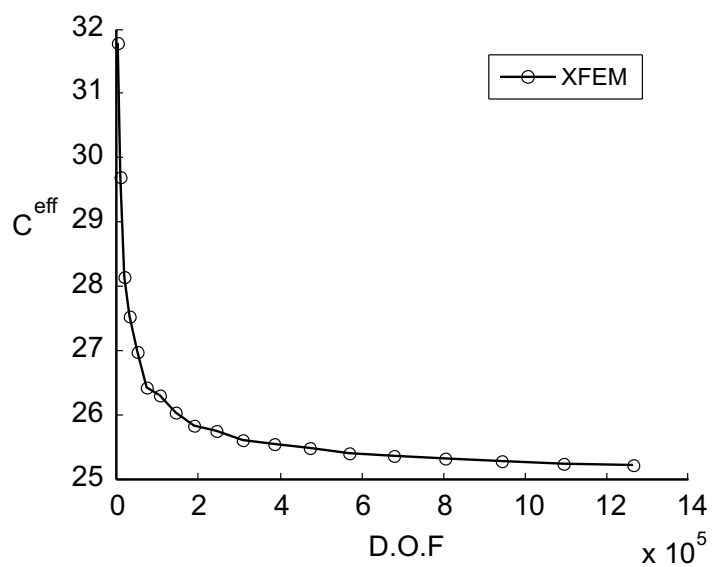

(b)

Fig. 9. a) Cubic containing 40 random spherical overlapped coated inclusions; b) Convergence of the effective conductivity versus the total number of d.o.f. 
National Laboratories, who reports in [22] that common industrial experience attributes $80 \%$ of the overall analysis time to the transfer of a complex geometric model into an analysis suitable discretization and only $20 \%$ to finite element analysis itself. More complex geometric model requires more time to create the conforming mesh. Furthermore, in case of $r_{1}<0.85 r_{2}$ in the second example, while XFEM gives good results with regular mesh, Ansys can not create the conforming mesh for finite element analysis because of too thin interfacial domain. In the third example, we create a random overlapped coated inclusion (Fig. 9(a)) by changing only radius of some inclusions in the second test. The minimal and maximal exterior radii are given by $r_{2}^{\max }=0.3250 \mathrm{~L}$ and $r_{2}^{\min }=0.13 \mathrm{~L}$. The proposed method works well while FFT can not treat overlapped domain of spheres and it is very difficult to create conforming FEM mesh for this case. The effective conductivity versus the total number of d.o.f. is graphically presented in Fig. 9(b).

\section{CONCLUSION}

We have shown in this paper a XFEM/level-set procedure which is suitable for the numerical computation of the effective thermal conductivities of coated composites. The proposed method that use multiple level-set functions to describe interfaces of coated inclusions and additional d.o.f. are introduced. The numerical examples show a excellent agreement between the proposed scheme and the FEM one. The proposed method can be extended to the case of multi-coated inclusions.

\section{ACKNOWLEDGEMENT}

This research is funded by Vietnam National Foundation for Science and Technology Development (NAFOSTED) under grant number 107.02-2014.08.

\section{REFERENCES}

[1] K. Suganuma, T. Okamoto, M. Koizljmi, and M. Shimada. Effect of interlayers in ceramicmetal joints with thermal expansion mismatches. Journal of the American Ceramic Society, 67, (12), (1984), pp. 256-257. doi:10.1111/j.1151-2916.1984.tb19688.x.

[2] G. Vörös and B. Pukánszky. Effect of a soft interlayer with changing properties on the stress distribution around inclusions and yielding of composites. Composites Part A: Applied Science and Manufacturing, 32, (3), (2001), pp. 343-352. doi:10.1016/s1359-835x(00)00125-1.

[3] L. J. Walpole. A coated inclusion in an elastic medium. In Mathematical Proceedings of the Cambridge Philosophical Society, Vol. 83. Cambridge Univ Press, (1978), pp. 495-506. doi:10.1017/s0305004100054773.

[4] Z. Hashin. The spherical inclusion with imperfect interface. Journal of Applied Mechanics, 58, (2), (1991), pp. 444-449. doi:10.1115/1.2897205.

[5] Y. P. Qiu and G. J. Weng. Elastic moduli of thickly coated particle and fiber-reinforced composites. Journal of Applied Mechanics, 58, (2), (1991), pp. 388-398. doi:10.1115/1.2897198.

[6] M. Hori and S. Nemat-Nasser. Double-inclusion model and overall moduli of multiphase composites. Mechanics of Materials, 14, (3), (1993), pp. 189-206. doi:10.1016/01676636(93)90066-z.

[7] M. Cherkaoui, H. Sabar, and M. Berveiller. Micromechanical approach of the coated inclusion problem and applications to composite materials. Journal of Engineering Materials and Technology, 116, (3), (1994), pp. 274-278. doi:10.1115/1.2904286. 
[8] M. Cherkaoui, H. Sabar, and M. Berveiller. Elastic composites with coated reinforcements: a micromechanical approach for nonhomothetic topology. International Journal of Engineering Science, 33, (6), (1995), pp. 829-843. doi:10.1016/0020-7225(94)00108-v.

[9] M. Cherkaoui, D. Muller, H. Sabar, and M. Berveiller. Thermoelastic behavior of composites with coated reinforcements: a micromechanical approach and applications. Computational Materials Science, 5, (1), (1996), pp. 45-52. doi:10.1016/0927-0256(95)00057-7.

[10] D. C. Pham and B. V. Tran. Equivalent-inclusion approach and effective medium approximations for conductivity of coated-inclusion composites. European Journal of Mechanics-A/Solids, 47, (2014), pp. 341-348. doi:10.1016/j.euromechsol.2014.05.010.

[11] H. Berger, S. Kurukuri, S. Kari, U. Gabbert, R. Rodriguez-Ramos, J. Bravo-Castillero, and R. Guinovart-Diaz. Numerical and analytical approaches for calculating the effective thermo-mechanical properties of three-phase composites. Journal of Thermal Stresses, 30, (8), (2007), pp. 801-817. doi:10.1080/01495730701415665.

[12] H. Y. Kuo and C. Y. Peng. Magnetoelectricity in coated fibrous composites of piezoelectric and piezomagnetic phases. International Journal of Engineering Science, 62, (2013), pp. 70-83. doi:10.1016/j.ijengsci.2012.08.002.

[13] F. T. Fisher and L. C. Brinson. Viscoelastic interphases in polymer-matrix composites: theoretical models and finite-element analysis. Composites Science and Technology, 61, (5), (2001), pp. 731-748. doi:10.1016/s0266-3538(01)00002-1.

[14] T. K. Nguyen and D. C. Pham. Equivalent-inclusion approach and effective medium estimates for elastic moduli of two-dimensional suspensions of compound inclusions. Philosophical Magazine, 94, (36), (2014), pp. 4138-4156. doi:10.1080/14786435.2014.978831.

[15] N. V. Luat and N. T. Kien. FFT simulations and multi-coated inclusion model for macroscopic conductivity of 2D suspensions of compound inclusions. Vietnam Journal of Mechanics, 37, (3), (2015), pp. 169-176. doi:10.15625/0866-7136/37/3/5096.

[16] E. Benvenuti. XFEM with equivalent eigenstrain for matrix-inclusion interfaces. Computational Mechanics, 53, (5), (2014), pp. 893-908. doi:10.1007/s00466-013-0938-4.

[17] E. Benvenuti, G. Ventura, N. Ponara, and A. Tralli. Variationally consistent eXtended FE model for 3D planar and curved imperfect interfaces. Computer Methods in Applied Mechanics and Engineering, 267, (2013), pp. 434-457. doi:10.1016/j.cma.2013.08.013.

[18] Q. Z. Zhu, S. T. Gu, J. Yvonnet, J. F. Shao, and Q. C. He. Three-dimensional numerical modelling by XFEM of spring-layer imperfect curved interfaces with applications to linearly elastic composite materials. International Journal for Numerical Methods in Engineering, 88, (4), (2011), pp. 307-328. doi:10.1002/nme.3175.

[19] S. T. Gu and Q. C. He. Interfacial discontinuity relations for coupled multifield phenomena and their application to the modeling of thin interphases as imperfect interfaces. Journal of the Mechanics and Physics of Solids, 59, (7), (2011), pp. 1413-1426. doi:10.1016/j.jmps.2011.04.004.

[20] J. Yvonnet, Q. C. He, and C. Toulemonde. Numerical modelling of the effective conductivities of composites with arbitrarily shaped inclusions and highly conducting interface. Composites Science and Technology, 68, (13), (2008), pp. 2818-2825. doi:10.1016/j.compscitech.2008.06.008.

[21] A. B. Tran, J. Yvonnet, Q. C. He, C. Toulemonde, and J. Sanahuja. A multiple level set approach to prevent numerical artefacts in complex microstructures with nearby inclusions within XFEM. International Journal for Numerical Methods in Engineering, 85, (11), (2011), pp. 1436-1459. doi:10.1002/nme.3025.

[22] J. A. Cottrell, T. J. R. Hughes, and Y. Bazilevs. Isogeometric analysis: toward integration of CAD and FEA. John Wiley \& Sons, (2009). 